\title{
Semi-perfect obstruction theory and Donaldson-Thomas invariants of derived objects
}

\author{
HuAi-Liang Chang AND Jun Li
}

\begin{abstract}
We introduce a semi-perfect obstruction theory of a DeligneMumford stack $X$ that consists of local perfect obstruction theories with a global obstruction sheaf. We construct the virtual cycle of a Deligne-Mumford stack with a semi-perfect obstruction theory. We use semi-perfect obstruction theory to construct virtual cycles of moduli of derived objects on Calabi-Yau threefolds.
\end{abstract}

\section{Introduction}

In this note, we introduce the notion of semi-perfect obstruction of a DeligneMumford stack. This notion has the advantage of the two perfect obstruction theories introduced in [2,13], by combining the local version of the perfect obstruction theory formulated in [2] with the locality of the virtual normal cone proved in [13]. This construction applies to moduli spaces that do not have universal families, like moduli of derived objects; it makes working with virtual cycles flexible.

Let $X \rightarrow \mathcal{M}$ be a representable morphism from a Deligne-Mumford stack to a smooth Artin stack of pure dimension. A semi-perfect relative obstruction theory $\phi$ of $X \rightarrow \mathcal{M}$ consists of an étale cover $U_{\alpha} \rightarrow X$ and (truncated) perfect obstruction theory $\phi_{\alpha}: E_{\alpha} \rightarrow \mathbb{L}_{U_{\alpha} / \mathcal{M}}^{\bullet \geq-1}$ such that the obstruction sheaves $\mathcal{O} b_{\phi_{\alpha}}=H^{1}\left(E_{\alpha}^{\vee}\right)$ descend to an obstruction sheaf on $X$, and that the infinitesimal obstruction assignments of deforming closed points in $X$ are independent of the charts $U_{\alpha}$.

We denote by $\mathcal{O} b_{\phi}$ the sheaf stack of the obstruction sheaf $\mathcal{O} b_{\phi}$ of the semi-obstruction theory $\phi$. We let $s$ be the zero section of $\mathcal{O} b_{\phi}$. We will make sense of the group of cycles $Z_{*} \mathcal{O} b_{\phi}$, and construct a Gysin map $s^{!}: Z_{*} \mathcal{O} b_{\phi} \rightarrow$ $A_{*} X$.

Theorem 1.1. Suppose $X \rightarrow \mathcal{M}$ as stated has a semi-perfect relative obstruction theory $\phi$. Then the collection of intrisic normal cones $\mathcal{C}_{U_{\alpha} / \mathcal{M}} \subset$ 
$\left.h^{1} / h^{0}\left(\left(\mathbb{L}_{U_{\alpha} / \mathcal{M}}\right)^{\vee}\right)\right)$ push-forward and glue to a cycle $\left[\mathfrak{c}_{X / \mathcal{M}}\right] \in Z_{*} \mathcal{O} b_{\phi}$. We define the virtual class of $(X, \phi)$ be

$$
[X, \phi]^{\operatorname{vir}}=s^{!}\left[\mathfrak{c}_{X / \mathcal{M}}\right] \in A_{*} X
$$

This class has the usual properties satisfied by virtual classes.

Using the construction of the moduli spaces of derived objects by Inaba [5] and Lieblich [14], and the perfect obstruction of derived objects over Calabi-Yau threefolds by Huybrecht-Thomas [3], we show that the moduli of derived objects has a semi-perfect obstruction theory. Applying the main theorem, we obtain its virtual cycle, whose degree is the DonaldsonThomas invariant of the moduli space, and its deformation invariance property.

The new input of this paper is that we put the cycle associated to the coarse moduli of the intrinsic normal cone of a Deligne-Mumford stack into its obstruction sheaf, assuming the existence of a semi-perfect obstruction theory. Originally, Tian and the second named author [13] constructed the virtual cycle after constructing the virtual normal cone in a vector bundle that surjects onto the obstruction sheaf, and Behrend and Fantechi [2] constructed the virtual cycle by constructing the intrinsic normal cone in the normal sheaf of the stack and putting it in the bundle-stack $h^{1} / h^{0}$ of the obstruction theory. After [9] (see also [8] and [16]), one can apply the Gysin map to a cycle in the bundle-stack directly, bypassing the need of the mentioned global vector bundles. Later, in $[11,12]$ the intrinsic normal cone was implicitly put inside the obstruction sheaf. For moduli spaces that lack universal families but do have obstruction sheaves (of their semiperfect obstruction theories), putting their intrinsic normal cones in the obstruction sheaves allows one to apply the Gysin map to obtain their virtual cycles.

Convention. In this paper, for a morphism $\rho: V \rightarrow U$ that is either a closed embedding, an étale morphism, or a composition of both, we will use restricting to $V$, i.e. $\left.\cdot\right|_{V}$, to denote the pullback under $\rho$. For instance, given a derive object $E$ on $U$, we denote by $\left.E\right|_{V}=\rho^{*} E$; for a stack $Y$ over $U$, we denote by $\left.Y\right|_{V}=Y \times_{U} V$, and for $\phi: E \rightarrow F$ a homomorphism of derived objects on $U$, we denote by $\left.\phi\right|_{V}$ the pullback homomorphism $\rho^{*} \phi: \rho^{*} E \rightarrow \rho^{*} F$.

We fix a characteristic zero algebraically closed field $\mathbf{k}$. All schemes and stacks in this paper are defined over $\mathbf{k}$. 


\section{Intrinsic normal cones in the obstruction sheaves}

In this section, we put the intrinsic normal cone in the obstruction sheaf, and show that it only depend on the the obstruction class assignments.

We let $\mathcal{M}$ be as before, which is a pure dimension smooth Artin stack of finite presentations. Let $U \rightarrow \mathcal{M}$ be a morphism from a scheme of finite type to $\mathcal{M}$. In this paper, we denote by $D(U)$ the derived complex of coherent sheaves of $\mathcal{O}_{U}$-modules; we denote by $\mathbb{L}_{U / \mathcal{M}}$ the relative cotangent complex of $U \rightarrow \mathcal{M}[4]$, and by $L_{U / \mathcal{M}}$ its truncation $L_{U / \mathcal{M}}=\mathbb{L}_{U / \mathcal{M}}^{\geq-1}$.

Definition 2.1 [2]. A (truncated) perfect (relative) obstruction theory of $U \rightarrow \mathcal{M}$ consists of a morphism in $D(U)$

$$
\phi: E \longrightarrow L_{U / \mathcal{M}}
$$

such that

(1) $E$ is a perfect complex in $D(U)$ of amplitude contained in $[-1,0]$;

(2) $h^{0}(\phi)$ is an isomorphism, and $h^{-1}(\phi)$ is surjective.

Given $\phi$, we call $\mathcal{O} b_{\phi}:=h^{1}\left(E^{\vee}\right)$ its obstruction sheaf.

For a derive object $B \in D(U)$ quasi-isomorphic to a complex of locally free sheaves $\left[B_{0} \longrightarrow B_{1} \stackrel{d_{1}}{\longrightarrow} \cdots\right]$, we define $h^{1} / h^{0}(B)$ to be the quotient stack $\left[\operatorname{ker}\left(d_{1}\right) / B_{0}\right][2]$. Following the authors in $[2,9]$, we denote the intrinsic normal cone and the intrinsic normal sheaf of $U / \mathcal{M}$ by

$$
\mathcal{C}_{U / \mathcal{M}} \subset N_{U / \mathcal{M}}:=h^{1} / h^{0}\left(\left(L_{U / \mathcal{M}}\right)^{\vee}\right) \cong h^{1} / h^{0}\left(\left(\mathbb{L}_{U / \mathcal{M}}\right)^{\vee}\right),
$$

we denote its associated cycle by $\left[\mathcal{C}_{U / \mathcal{M}}\right] \in Z_{*} N_{U / \mathcal{M}}$. Let

$$
h^{1} / h^{0}\left(\phi^{\vee}\right): N_{U / \mathcal{M}} \stackrel{\subset}{\longrightarrow} h^{1} / h^{0}\left(E^{\vee}\right)
$$

be induced by the truncated perfect obstruction theory.

In this paper, for a coherent sheaf $\mathcal{F}$ of $\mathcal{O}_{U}$-modules. We form the sheaf stack of $\mathcal{F}$, which is the groupoid that associates to any $\rho: S \rightarrow U$ the set $\Gamma\left(S, \rho^{*} \mathcal{F}\right)$. By abuse of notation, we denote the sheaf stack of $\mathcal{F}$ by the same symbol $\mathcal{F}$. And following the $h^{1} / h^{0}$ notation, we denote by $h^{1}\left(E^{\vee}\right)$ the sheaf stack of the cohomology sheaf $H^{1}\left(E^{\vee}\right)$. Applying this construction to $\mathcal{O} b_{\phi}=H^{1}\left(E^{\vee}\right)$ and composed with (2.3), we have the induced morphism 
of stacks

$$
\eta_{\phi}: N_{U / \mathcal{M}} \longrightarrow h^{1} / h^{0}\left(E^{\vee}\right) \longrightarrow h^{1}\left(E^{\vee}\right)
$$

where the last arrow is defined as follows.

We claim that given a complex of locally free sheaves $F=\left[\mathcal{F}_{0} \rightarrow \mathcal{F}_{1}\right]$ on $U$, the homomorphism of sheaves $\mathcal{F}_{1} \rightarrow H^{1}(F)$ induces a tautological morphism

$$
h^{1} / h^{0}(F) \longrightarrow h^{1}(F)
$$

of stacks. Indeed, given any morphism $\rho: S \rightarrow U$, an element in $h^{1} / h^{0}(F)(S)$ consists of a principal $G=F_{0} \times_{U} S$-bundle $R$, where $F_{i}$ is the associated vector bundle of $\mathcal{F}_{i}$ (i.e. $\mathcal{O}_{U}\left(F_{i}\right)=\mathcal{F}_{i}$ ) and $G$ is viewed as an $S$-group scheme, and a $G$-equivariant $S$-morphism $\zeta: R \rightarrow F_{1} \times_{U} S$, where $G$ acts on $F_{1} \times_{U} S$ via the linear homomorphism $F_{0} \rightarrow F_{1}$. Equivalently, $\zeta \in \Gamma\left(R, \mathcal{F}_{1} \otimes_{\mathcal{O}_{U}} \mathcal{O}_{R}\right)$. Let $q: \mathcal{F}_{1} \rightarrow H^{1}(F)$ be the projection. Then $q \circ \zeta \in \Gamma\left(R, H^{1}(F) \otimes_{\mathcal{O}_{U}} \mathcal{O}_{R}\right)$. Since $\zeta$ is $G$-equivariant, and since the induced $G$-action on $H^{1}(F) \otimes_{\mathcal{O}_{U}} \mathcal{O}_{R}$ is trivial, $q \circ \zeta$ descends to a section $\tilde{\zeta} \in \Gamma\left(S, H^{1}(F) \otimes \mathcal{O}_{U} \mathcal{O}_{S}\right)$, which is an element in $h^{1}(F)(S)$. Since this correspondence $\zeta \mapsto \tilde{\zeta}$ is canonical, it defines a desired morphism (2.5).

Definition 2.2. We call a substack $A \subset \mathcal{F}$ a reduced cycle if for any locally free sheaf $\mathcal{V}$ of $\mathcal{O}_{U}$-modules and a surjective $f: \mathcal{V} \rightarrow \mathcal{F}$, and denoting by the same $f$ the induced morphism of their respective stacks $\mathcal{V} \rightarrow \mathcal{F},(\mathcal{V}$ as the total space of the vector bundle $\mathcal{V},) \mathcal{V} \times_{\mathcal{F}} A$ is a reduced Zariski closed subset of $\mathcal{V}$. We say $A$ is integral if it is reduced and is not a union of two distinct non-empty reduced cycle of $\mathcal{F}$. We define $Z_{*} \mathcal{F}$ to be the (rational) linear combinations of integral cycles in $\mathcal{F}$.

Lemma 2.1. Let $A \subset h^{1} / h^{0}\left(E^{\vee}\right)$ be an integral cycle. Then the image $\eta_{\phi}(A) \subset h^{1}\left(E^{\vee}\right)$ is an integral cycle in $h^{1}\left(E^{\vee}\right)$. We call the cycle $\left[\eta_{\phi}(A)\right]$ the push-forward of $[A]$, and denote it by $\eta_{\phi *}[A] \in Z_{*} h^{1}\left(E^{\vee}\right)$.

Proof. We let $E=\left[E_{-1} \rightarrow E_{0}\right]$ with both $E_{i}$ locally free; let $\mathcal{V}=E_{-1}^{\vee}$ and let $\mathcal{V}[-1] \rightarrow E^{\vee}$ be induced by the identity $\mathcal{V} \rightarrow E_{-1}^{\vee}$. By viewing $\mathcal{V}$ as the sheaf stack of $\mathcal{V}$, the arrow $\mathcal{V}[-1] \rightarrow E^{\vee}$ induces a morphism $p: \mathcal{V} \rightarrow h^{1} / h^{0}\left(E^{\vee}\right)$.

Let $A \subset h^{1} / h^{0}\left(E^{\vee}\right)$ be an integral Artin substack. By definition,

$$
p^{-1}(A):=A \times_{h^{1} / h^{0}\left(E^{\vee}\right)} \mathcal{V} \subset \mathcal{V}
$$


is a reduced Zariski closed subset. Let $p^{\prime}: \mathcal{V} \rightarrow h^{1}\left(E^{\vee}\right)$ be the morphism of stack induced by the same $\mathcal{V}[-1] \rightarrow E^{\vee}$. It fits into a commutative square

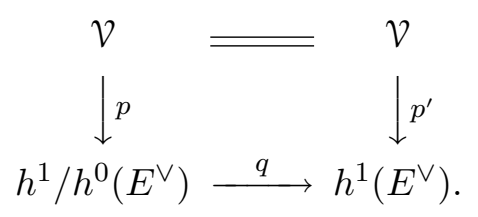

We claim that

$$
p^{\prime-1}(q(A)):=q(A) \times_{h^{1}\left(E^{\vee}\right)} \mathcal{V}=p^{-1}(A) \subset \mathcal{V} .
$$

We prove that $p^{-1}(q(A)) \subset p^{-1}(A)$; the other direction of inclusion is similar and more direct. Let $T$ be any affine scheme and $\rho: T \rightarrow U$ a morphism. By definition, an object in $p^{\prime-1}(q(A))(\rho)$ consists of a pair of a $T$-morphism $\xi_{1}:\left.T \rightarrow \mathcal{V}\right|_{T}$ and an $\left.E_{0}^{\vee}\right|_{T}$-equivariant $T$-morphism $\xi_{2}: P \rightarrow$

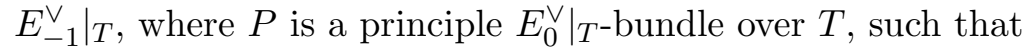

$$
p^{\prime} \circ \xi_{1}=q \circ \xi_{2} / E_{0}^{\vee},
$$

where $q \circ \xi_{2} / E_{0}^{\vee}$ is the descent of $q \circ \xi_{2}$ to $\left.T \rightarrow h^{1}\left(E^{\vee}\right)\right|_{T}$ using that $E_{0}^{\vee}$ acts on $h^{1}\left(E^{\vee}\right)$ trivially.

We need to show that $\xi_{1}$ is an object in $p^{-1}(A)(\rho)$. For this, it suffices to show that $p\left(\xi_{1}\right) \cong \xi_{2}$. Following [2, Sect. 2], $p\left(\xi_{1}\right)$ is given by the $\left.E_{0}^{\vee}\right|_{T^{-}}$ principle bundle $P^{\prime}=\left.E_{0}^{\vee}\right|_{T}$ with the tautological projection $\pi^{\prime}: P^{\prime} \rightarrow T$ and a $\xi_{2}^{\prime}:\left.P^{\prime} \rightarrow E_{-1}^{\vee}\right|_{T}$ via $\xi_{2}^{\prime}(v)=d^{\vee}(v)+\xi_{1}\left(\pi^{\prime}(v)\right)$, where $d^{\vee}: E_{0}^{\vee} \rightarrow E_{-1}^{\vee}$ is the dual of $\left[E_{-1} \rightarrow E_{0}\right]$.

Because $T$ is affine, we can find a section $s: T \rightarrow P$ of the bundle $P \rightarrow T$. Then (2.6) implies that

$$
p^{\prime} \circ \xi_{1}=(q \circ p) \circ \xi_{2} \circ s=p^{\prime} \circ \xi_{2} \circ s:\left.T \longrightarrow h^{1}\left(E^{\vee}\right)\right|_{T} .
$$

Therefore, there is a section $t:\left.T \rightarrow E_{0}^{\vee}\right|_{T}$ so that $\xi_{2} \circ s=\xi_{1}+d^{\vee} \circ t: T \rightarrow$ $\mathcal{V}$. Therefore, we can find an isomorphism $P \cong P^{\prime}$ as principle bundle so that $\xi_{2}^{\prime} \cong \xi_{2}$. This proves that $p\left(\xi_{1}\right) \cong \xi_{2}$; namely, $\xi_{1}$ is an object in $p^{-1}(A)$. This proves the lemma.

Representing $\left[\mathcal{C}_{U / \mathcal{M}}\right]$ as a linear combination of integral cycles in $N_{U / \mathcal{M}}$, and applying the push-forward $\eta_{\phi *}$, we obtain

$$
\left[\mathfrak{c}_{\phi}\right]=\eta_{\phi *}\left[\mathcal{C}_{U / \mathcal{M}}\right] \in Z_{*} h^{1}\left(E^{\vee}\right) .
$$


In the remainder of this subsection, we study the dependence of this cycle on the obstruction theory $\phi$. Suppose we have another truncated perfect relative obstruction theory

$$
\phi^{\prime}: E^{\prime} \longrightarrow L_{U / \mathcal{M}}
$$

and suppose we have an isomorphism

$$
\psi: h^{1}\left(E^{\vee}\right) \stackrel{\cong}{\longrightarrow} h^{1}\left(E^{\prime \vee}\right) .
$$

We study when the cycles $\psi_{*}\left[\mathfrak{c}_{\phi}\right]=\left[\mathfrak{c}_{\phi^{\prime}}\right]$.

As was proved in [13], the cycle $\left[\mathfrak{c}_{\phi}\right]$ is determined by the obstruction theories to deforming closed points in $X$. The uniqueness proof given here reminiscent to that in [13].

For any closed $p \in U$, we denote

$$
T_{p, U / \mathcal{M}}^{i}=H^{i}\left(\left.\left(L_{U / \mathcal{M}}\right)^{\vee}\right|_{p}\right)
$$

Definition 2.3. We define the intrinsic obstruction space to deforming $p \in U$ to be $T_{p, U / \mathcal{M}}^{1}$; we define the obstruction space (of the obstruction theory $\phi)$ to deforming $p \in U$ to be $\mathrm{Ob}(\phi, p)=H^{1}\left(\left.E^{\vee}\right|_{p}\right)$.

Definition 2.4. Let $\iota: T \rightarrow T^{\prime}$ be a closed subscheme with $T^{\prime}$ local Artinian. Let $I$ be the ideal sheaf of $T$ in $T^{\prime}$, and let $\mathfrak{m}$ be the ideal sheaf of the closed point of $T^{\prime}$. We call $\iota$ a small extension if $I \cdot \mathfrak{m}=0$. Given a small extension $\left(T, T^{\prime}, I, \mathfrak{m}\right)$ that fits into a commutative square

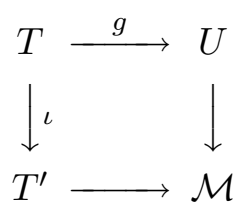

so that the image of $g$ contains a closed point $p \in U$, finding a morphism $g^{\prime}: T^{\prime} \rightarrow U$ that commutes with the arrows in (2.10) is called "infinitesimal lifting problem of $U / \mathcal{M}$ at $p "$.

Applying the standard obstruction theory and using

$$
\operatorname{Ext}^{i}\left(g^{*} L_{U / \mathcal{M}}, I\right)=\operatorname{Ext}^{i}\left(g^{*} \mathbb{L}_{U / \mathcal{M}}, I\right), \quad i=0,1,
$$

we obtain 
Lemma 2.2 [4, Chap. 3, Thm. 2.1.7]. For an infinitesimal lifting problem of $U / \mathcal{M}$ at $p$ as in (2.10), there is a canonical element

$$
\omega\left(g, T, T^{\prime}\right) \in \operatorname{Ext}^{1}\left(g^{*} L_{U / \mathcal{M}}, I\right)=T_{p, U / \mathcal{M}}^{1} \otimes_{\mathbf{k}} I,
$$

whose vanishing is necessary and sufficient for the lifting problem to be solvable; in case the lifting problem is solvable, the collection of the solutions form a torsor under

$$
\operatorname{Ext}^{0}\left(g^{*} L_{U / \mathcal{M}}, I\right)=\operatorname{Hom}\left(g^{*} \Omega_{U / \mathcal{M}}, I\right)
$$

Definition 2.5. Let $\phi$ in (2.1) be a perfect obstruction theory. For the infinitesimal lifting problem (2.10), we call the image

$$
\mathrm{ob}\left(\phi, g, T, T^{\prime}\right):=H^{1}\left(\phi^{\vee}\right)\left(\omega\left(g, T, T^{\prime}\right)\right) \in \operatorname{Ext}^{1}\left(g^{*} E, I\right)=\mathrm{Ob}(\phi, p) \otimes_{\mathbf{k}} I
$$

the obstruction class (of $\phi$ ) to the lifting problem (2.10).

Corollary 2.1 [2]. Let $\phi$ in (2.1) be a perfect relative obstruction theory. Then the lifting problem (2.10) is solvable if and only if ob( $\left.\phi, g, T, T^{\prime}\right)=0$.

Proof. This is true because $H^{1}\left(\left.\phi^{\vee}\right|_{p}\right)$ is injective.

We now back to the pair of obstruction theories $\phi$ and $\phi^{\prime}$ mentioned in $(2.7)$.

Definition 2.6. We call $\phi$ and $\phi^{\prime} \nu$-equivalent if there is an isomorphism of sheaves

$$
\psi: H^{1}\left(E^{\vee}\right) \stackrel{\cong}{\longrightarrow} H^{1}\left(E^{\prime \vee}\right)
$$

so that for every closed point $p \in U$, and for any "infinitesimal lifting problem of $U / \mathcal{M}$ at $p "$ as in (2.10), we have

$$
\left.\psi\right|_{p}\left(\mathrm{ob}\left(\phi, g, T, T^{\prime}\right)\right)=\mathrm{ob}\left(\phi^{\prime}, g, T, T^{\prime}\right) \in \mathrm{Ob}\left(\phi^{\prime}, p\right) \otimes_{\mathbf{k}} I .
$$

The main result of this section is

Proposition 2.1. Let (2.13) be a $\nu$-equivalence of $\phi$ and $\phi^{\prime}$, and let $\eta_{\phi}$ : $N_{U / \mathcal{M}} \rightarrow h^{1}\left(E^{\vee}\right)$ be the induced morphisms of stacks (2.4). Then for any integral cycle $A \subset N_{U / \mathcal{M}}$,

$$
\psi_{*}\left(\eta_{\phi *}[A]\right)=\eta_{\phi^{\prime} *}[A] \in Z_{*} h^{1}\left(E^{\prime \vee}\right) .
$$


We recall the following known facts.

Lemma 2.3. Suppose $G$ is a perfect complex over $U$ of amplitude $\leq 0$. Let $p \in U$ be a closed point and denote $V^{i}=H^{i}\left(\left.G^{\vee}\right|_{p}\right)$. Then canonically, $h^{1} /\left.h^{0}\left(G^{\vee}\right)\right|_{p} \cong\left[V^{1} / V^{0}\right]$, where $V^{0}$ acts on $V^{1}$ trivially.

Proof. Let $G=\left[\cdots \stackrel{d_{-1}}{\longrightarrow} G_{-1} \stackrel{d_{0}}{\longrightarrow} G_{0} \rightarrow 0\right]$, where $G_{i}$ are locally free. Without loss of generality, we assume $\left.d_{0}\right|_{p}:\left.\left.G_{-1}\right|_{p} \rightarrow G_{0}\right|_{p}$ is trivial. Let $G_{-1}^{\prime}=$ $\operatorname{coker}\left(d_{-1}\right)$. Then $G^{\geq-1}=\left[G_{-1}^{\prime} \rightarrow G_{0}\right]$. We let $C=\operatorname{Spec} \operatorname{Sym}\left(G_{-1}^{\prime}\right)$. Then the arrow $G_{-1}^{\prime} \rightarrow G_{0}$ defines an action $G_{0}^{\vee} \times_{Y} C \rightarrow C$, where we view $G_{0}^{\vee}$ as the total space of the bundle $G_{0}^{\vee}$. By definition $[2], h^{1} / h^{0}\left(G^{\vee}\right)=\left[C / G_{0}^{\vee}\right]$, where $[\cdot]$ means the quotient stack.

By the base change property of $\operatorname{coker}\left(d_{-1}\right)$, we have $\operatorname{coker}\left(\left.d_{-1}\right|_{p}\right)=$ $\left.G_{-1}^{\prime}\right|_{p}$. Since $\left.d_{0}\right|_{p}=0$, we have $C \times_{U} p=V^{1}$. The lemma then follows from the definition of $h^{1} / h^{0}$ construction.

We quote the following:

Lemma 2.4 [2, Prop. 4.7]. Let the situation be as in Definition 2.4, and let $p$ be a closed point of $U$. Given any vector $v \in T_{p, U / \mathcal{M}}^{1}$, there exists an infinitesimal lifting problem of $U / \mathcal{M}$ at $p$ as in Definition 2.4 such that for some $w \neq 0 \in I$, the obstruction class

$$
w\left(g, T, T^{\prime}\right)=v \otimes w \in \operatorname{Ext}^{1}\left(g^{*} L_{U / \mathcal{M}}, I\right) \cong T_{p, U / \mathcal{M}}^{1} \otimes_{\mathbf{k}(p)} I
$$

Proof of Proposition 2.1. Let $E^{\prime}=\left[E_{-1}^{\prime} \rightarrow E_{0}^{\prime}\right]$ with both $E_{i}^{\prime}$ locally free. Let $V=E_{-1}^{\prime \vee}$. To prove the proposition, by definition, we need to show that for the surjective morphism $\mathcal{V} \rightarrow h^{1}\left(E^{\prime \vee}\right)$,

$$
\psi\left(\eta_{\phi}(A)\right) \times_{h^{1}\left(E^{\prime \vee}\right)} \mathcal{V}=\eta_{\phi^{\prime}}(A) \times_{h^{1}\left(E^{\prime \vee}\right)} \mathcal{V}
$$

as subsets in $\mathcal{V}$. Since $\mathcal{V}$ is a scheme and $A$ is integral, both sides of (2.14) are reduced and Zariski closed. Thus to show (2.14), it suffices to check that for any closed $p \in U$, we have

$$
\psi\left(\eta_{\phi}(A)\right) \times_{h^{1}\left(E^{\prime \vee}\right)}\left(\left.\mathcal{V}\right|_{p}\right)=\eta_{\phi^{\prime}}(A) \times_{h^{1}\left(E^{\prime \vee}\right)}\left(\left.\mathcal{V}\right|_{p}\right) .
$$


But this follows from that there is an isomorphism $\tau_{p}$ making the following square commutative:

$$
\begin{aligned}
& \left.N_{U / \mathcal{M}}\right|_{p}:=h^{1} /\left.\left.h^{0}\left(L_{U / \mathcal{M}}^{\vee}\right)\right|_{p} \stackrel{\left.\eta_{\phi}\right|_{p}}{\longrightarrow} h^{1}\left(E^{\vee}\right)\right|_{p} \\
& \left.\downarrow \tau_{p} \quad \downarrow \psi\right|_{p} \\
& \left.N_{U / \mathcal{M}}\right|_{p}:=h^{1} /\left.\left.h^{0}\left(L_{U / \mathcal{M}}^{\vee}\right)\right|_{p} \stackrel{\left.\eta_{\phi^{\prime}}\right|_{p}}{\longrightarrow} h^{1}\left(E^{\prime \vee}\right)\right|_{p} \text {. }
\end{aligned}
$$

Applying Lemma to $G=L_{U / M}$, we obtain canonical isomorphisms

$$
h^{1} /\left.h^{0}\left(\left(L_{U / \mathcal{M}}\right)^{\vee}\right)\right|_{p} \cong\left[H^{1}\left(\left.L_{U / \mathcal{M}}\right|_{p}\right)^{\vee} / H^{0}\left(\left.L_{U / \mathcal{M}}\right|_{p}\right)^{\vee}\right]=\left[T_{p, U / \mathcal{M}}^{1} / T_{p, U / \mathcal{M}}^{0}\right]
$$

applying the same lemma to $G=E$, we obtain the identity $\left.h^{1}\left(E^{\vee}\right)\right|_{p}=$ $\mathrm{Ob}(\phi, p)$. Similarly we obtain isomorphisms with $\phi$ and $E$ replaced by $\phi^{\prime}$ and $E^{\prime}$. Therefore, the existence of $\tau_{p}$ making (2.15) commutative if the square

$$
\begin{array}{cr}
T_{p, U / \mathcal{M}}^{1} \stackrel{H^{1}\left(\left.\phi^{\vee}\right|_{p}\right)}{\longrightarrow} & \mathrm{Ob}(\phi, p) \\
\| & \left.\downarrow \psi\right|_{p} \\
T_{p, U / \mathcal{M}}^{1} \stackrel{H^{1}\left(\left.\phi^{\prime \vee}\right|_{p}\right)}{\longrightarrow} \mathrm{Ob}\left(\phi^{\prime}, p\right)
\end{array}
$$

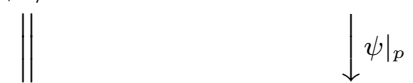

is commutative.

We prove this commutativity. Given any $v \in T_{p, U / \mathcal{M}}^{1}$, by Lemma 2.4 there exists an infinitesimal lifting problem $\left(g, T, T^{\prime}\right)$ for $U / \mathcal{M}$ at $p$ as in (2.10) and a $0 \neq w \in I$ such that

$$
v \otimes w=\omega\left(g, T, T^{\prime}\right) \in T_{p, U / \mathcal{M}}^{1} \otimes_{\mathbf{k}(p)} I
$$

Since $\phi$ and $\phi^{\prime}$ are numerically equivalent, we have

$$
\begin{aligned}
H^{1}\left(\left.\phi^{\vee}\right|_{p}\right)(v) \otimes w & =H^{1}\left(\left.\phi^{\vee}\right|_{p}\right)(v \otimes w) \\
& =H^{1}\left(\left.\psi^{\vee}\right|_{p}\right)\left(H^{1}\left(\left.\phi^{\prime \vee}\right|_{p}\right)(v \otimes w)\right) \\
& =H^{1}\left(\left.\psi^{\vee}\right|_{p}\right)\left(H^{1}\left(\left.\phi^{\prime \vee}\right|_{p}\right)(v)\right) \otimes w
\end{aligned}
$$

As $w \neq 0$ and $I$ is a $\mathbf{k}(p)$ vector space, we have

$$
H^{1}\left(\left.\phi^{\vee}\right|_{p}\right)(v)=H^{1}\left(\left.\psi^{\vee}\right|_{p}\right)\left(H^{1}\left(\left.\phi^{\prime \vee}\right|_{p}\right)(v)\right) \text {. }
$$

This proves the proposition. 


\section{Semi-perfect obstruction theory}

Let $\mathcal{M}$ be as before and let $X$ be a Deligne-Mumford stack of locally finite type with a morphism $X \rightarrow \mathcal{M}$. Given two schemes $U_{\alpha}$ and $U_{\beta}$ with étale $U_{\alpha}, U_{\beta} \rightarrow X$, we denote $U_{\alpha \beta}=U_{\alpha} \times_{X} U_{\beta}$, and for any derived object $F \in D\left(U_{\alpha}\right)$ we denote by $\left.F\right|_{U_{\alpha \beta}}$ the pull back of $F$ under the projection $U_{\alpha \beta} \rightarrow U_{\alpha}$.

Definition 3.1. A semi-perfect relative obstruction theory of $X \rightarrow \mathcal{M}$ consists of an étale covering $\left\{U_{\alpha}\right\}_{\alpha \in \Lambda}$ of $X$ by affine schemes, and truncated perfect relative obstruction theories

$$
\phi_{\alpha}: E_{\alpha} \longrightarrow L_{U_{\alpha} / \mathcal{M}}, \alpha \in \Lambda
$$

such that

1. for each pair $\alpha, \beta \in \Lambda$ there is an isomorphism

$$
\psi_{\alpha \beta}:\left.\left.H^{1}\left(E_{\alpha}^{\vee}\right)\right|_{U_{\alpha \beta}} \longrightarrow H^{1}\left(E_{\beta}^{\vee}\right)\right|_{U_{\alpha \beta}},
$$

so that the collection $\left(H^{1}\left(E_{\alpha}^{\vee}\right), \psi_{\alpha \beta}\right)$ forms a descent data of sheaves.

2. for any pair $\alpha, \beta \in \Lambda$, the obstruction theories $\left.\phi_{\alpha}\right|_{U_{\alpha \beta}}$ and $\left.\phi_{\beta}\right|_{U_{\alpha \beta}}$ are $\nu$-equivalent via $\psi_{\alpha \beta}$.

Obviously, a perfect obstruction theory is a semi-perfect obstruction theory.

We fix a semi-perfect obstruction theory $\phi=\left\{\phi_{\alpha}, U_{\alpha}, E_{\alpha}, \psi_{\alpha \beta}\right\}_{\Lambda}$. We denote by $\mathcal{O} b_{\phi}$ the resulting descent sheaf on $X$ from (1) of Definition 3.1; we call it the obstruction sheaf of the semi-perfect obstruction theory.

Let $\mathcal{F}$ be a coherent sheaf of $\mathcal{O}_{X}$-modules, viewed as a sheaf stack.

Definition 3.2. A reduced cycle $A$ of $\mathcal{F}$ is a substack $A \subset \mathcal{F}$ so that for any étale open $U \rightarrow X, U \times\left._{\mathcal{F}} A \subset \mathcal{F}\right|_{U}$ is a reduced cycle in the sense of Definition 2.2; we call $A$ integral if it is not a union of two distinct nontrivial reduced cycles. We define the cycle group $Z_{*} \mathcal{F}$ be (rational) linear combinations of integral cycles of $\mathcal{F}$.

Since $\mathcal{F}$ is a sheaf over $X$, by descent, $A \subset \mathcal{F}$ a reduced cycle if it is given by an étale covering $U_{\alpha} \rightarrow X$ and reduced cycles $\left.A_{\alpha} \subset \mathcal{F}\right|_{U_{\alpha}}$ such that 
over each $U_{\alpha \beta}$ :

$$
A_{\alpha} \times_{U_{\alpha}} U_{\alpha \beta}=A_{\beta} \times\left._{U_{\beta}} U_{\alpha \beta} \subset \mathcal{F}\right|_{U_{\alpha \beta}}
$$

We continue to work with the semi-perfect obstruction theory $\phi$ as in Definition 3.1. We construct a group homomorphism

$$
\eta_{*}: Z_{*} N_{X / \mathcal{M}} \longrightarrow Z_{*} \mathcal{O} b_{\phi}
$$

by patching the collection

$$
\eta_{\phi_{\alpha} *}: Z_{*} N_{U_{\alpha} / \mathcal{M}} \longrightarrow Z_{*} \mathcal{O} b_{\phi_{\alpha}} .
$$

Lemma 3.1. Given an integral Artin substack $[A] \subset Z_{*} N_{X / \mathcal{M}}$, the collection

$$
\left[A_{\alpha}\right]:=\left.\eta_{\phi_{\alpha} *}\left[A \times_{X} U_{\alpha}\right] \in Z_{*} \mathcal{O} b_{\phi}\right|_{U_{\alpha}}
$$

satisfies the descent condition (3.2) to form an integral cycle in $Z_{*} \mathcal{O} b_{\phi}$.

Proof. This follows from the definition of semi-perfect obstruction theories and Proposition 2.1.

We denote the resulting cycle by $\eta_{\phi *}[A]$. We denote by

$$
\eta_{\phi *}: Z_{*} N_{X / \mathcal{M}} \longrightarrow Z_{*} \mathcal{O} b_{\phi}
$$

the homomorphism by linear extension. Applying this to the cycle $\left[\mathcal{C}_{X / \mathcal{M}}\right] \in$ $Z_{*} N_{X / \mathcal{M}}$, we define

$$
\left[\mathfrak{c}_{X / \mathcal{M}}\right]=\eta_{\phi *}\left[\mathcal{C}_{X / \mathcal{M}}\right] \in Z_{*} \mathcal{O} b_{\phi}
$$

Let $s$ be the zero section of $\mathcal{O} b_{\phi}$. To define the virtual cycle of $X$, we need to construct a Gysin map $s^{!}: Z_{*} \mathcal{O} b_{\phi} \longrightarrow A_{*} X$. The Gysin map for a bundle stack is constructed in [9]. The construction given here was first introduced in the work of the second named author in [11]; it is reiterated in [6]. We now sketch its construction.

Assume $X$ is proper and of finite type. Let $\mathcal{F}$ be a coherent sheaf of $\mathcal{O}_{X}$ modules, considered as a sheaf stack.

Given a non-trivial integral cycle $A \subset \mathcal{F}$, where $\mathcal{F}$ is a sheaf stack of a coherent sheaf $\mathcal{F}$ of $\mathcal{O}_{X}$-modules, we pick an affine scheme $U$ and an étale 
$\rho: U \rightarrow X$ so that $A \times_{X} U \neq \emptyset$. Since $U$ is affine, we can find a vector bundle $\mathcal{V}_{U}$ on $U$ and a surjective $\left.\mathcal{V}_{U} \rightarrow \mathcal{F}\right|_{U}$. By definition,

$$
\left.A\right|_{U} \times\left.\right|_{\left.\right|_{U}} \mathcal{V}_{U} \subset \mathcal{V}_{U}
$$

is a reduced Zariski closed subset. We let $B \subset U$ be the image of $\left.A\right|_{U} \times \mathcal{F}_{U} \mathcal{V}_{U}$ under the projection $\mathcal{V}_{U} \rightarrow U$. We let $Y \subset X$ be the closure of the image $\rho(B)$. Because $A$ is integral, $Y$ is integral; because $X$ is proper, $Y$ is proper.

Because $X$ is a proper Deligne-Mumford stack, we can find a projective variety $S$ and a generic finite morphism $f: S \rightarrow Y$ [10, Cor. 16.6.1]. Then we can find a locally free sheaf $\mathcal{V}$ on $S$ and a surjective sheaf homomorphism $\mathcal{V} \rightarrow f^{*} \mathcal{F}$. Pick an open $S_{0} \subset S$ so that $\left.f\right|_{S_{0}}: S_{0} \rightarrow Y$ is étale. Let $D_{0}=$ $\left.\mathcal{V}\right|_{S_{0}} \times\left.\mathcal{F} A \subset \mathcal{V}\right|_{S_{0}}$, and let $D_{A} \subset \mathcal{V}$ be the closure of $D_{0}$. We call

$$
\left(f: S \rightarrow Y, \mathcal{V} \rightarrow f^{*} \mathcal{F}, D_{A} \subset \mathcal{V}\right)
$$

a proper representative of the integral cycle $A$. Let $e$ be the degree of $f$ : $S \rightarrow Y$. We define

$$
s^{!}[A]=e^{-1} f_{*}\left(0_{\mathcal{V}}^{!}\left[D_{A}\right]\right) \in A_{*} X,
$$

where $0 ! Z_{*}: \mathcal{V} \rightarrow A_{*} S$ is the Gysin map of the zero section of $\mathcal{V}$.

Proposition 3.1. The stated procedure defines a Gysin map

$$
s^{!}: Z_{*} \mathcal{F} \longrightarrow A_{*} X
$$

by linear extension.

Proof. We need to show that the map $s^{!}([A])$ of an integral $A \in Z_{*} \mathcal{F}$ is independent of the choice of the proper representatives of $A$. This is essentially proved in [11] and [6, Sect. 3]. We outline the main idea here. Let $\left(f^{\prime}, S^{\prime}, \mathcal{V}^{\prime}, D_{A}^{\prime}\right)$ be another proper representative of $A$. Then we can find a third proper representative $\left(\bar{f}, \bar{S}, \overline{\mathcal{V}}, \bar{D}_{A}\right)$ that fits into the commutative squares

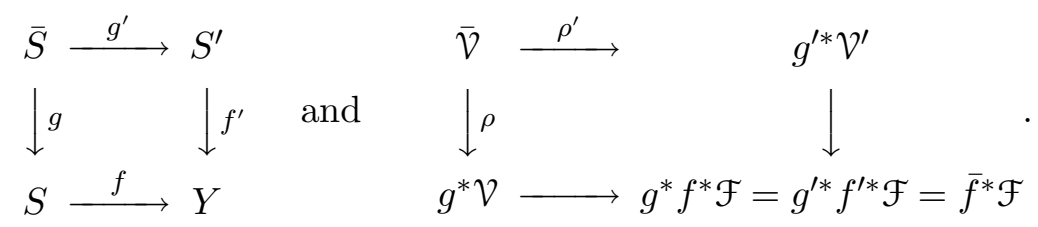

Since $\bar{f}$ is generically finite, both $g$ and $g^{\prime}$ are generically finite. Then by the construction of the cycles $D_{A}, D_{A}^{\prime}$ and $\bar{D}_{A} \subset \overline{\mathcal{V}}$, we have $\rho_{*}\left[\bar{D}_{A}\right]=d(g)\left[D_{A}\right]$ 
and $\rho_{*}^{\prime}\left[\bar{D}_{A}\right]=d\left(g^{\prime}\right)\left[D_{A}^{\prime}\right]$. Therefore, for $s^{\prime}$ and $\bar{s}$ the zero sections of $\mathcal{V}^{\prime}$ and $\bar{\nu}^{\prime}$, we have

$$
(\operatorname{deg} g) f_{*} s^{!}\left[D_{A}\right]=g_{*} \bar{s}^{!}\left[\bar{D}_{A}\right]=\left(\operatorname{deg} g^{\prime}\right) f_{*}^{\prime} s^{\prime}\left[D_{A}^{\prime}\right]
$$

Pushing forward to $A_{*} X$, we prove that (3.7) is well defined.

We prove that the Gysin map preserves the rational equivalence. Like the usual rational equivalence, an integral rational equivalence is a pair $(A, h)$ of an integral cycle $A$ of $\mathcal{F}$ and a non-trivial rational function on $A$, which we define now.

Definition 3.3. We define a proper representative of a rational function on an integral $A \in Z_{*} \mathcal{F}$ to be a proper representative $\left(f, \mathcal{V}, D_{A}\right)$ of $A$ as in (3.6) and a rational function $h_{f} \in \mathbf{k}\left(D_{A}\right)$ so that for $D_{A}^{\text {nor }}$ the normalization of $D_{A}, \tilde{h}_{f}$ the extension of $h_{f}$ to $D_{A}^{\text {nor }}$, and $\iota: D_{A}^{\text {nor }} \rightarrow \mathcal{F}$ the tautological morphism, we have that for any closed $p \in D_{A}^{\text {nor }}$, the restriction $\left.\tilde{h}_{f}\right|_{\iota^{-1}(\iota(p))}$ is either nowhere defined or takes a single value.

Let $\left(f^{\prime}, S^{\prime}, \mathcal{V}^{\prime}, D_{A}^{\prime}\right)$ and $h_{f^{\prime}}^{\prime} \in \mathbf{k}\left(D_{a}^{\prime}\right)$ be another proper representative of a rational function on $A$. We say $h_{f} \sim h_{f}^{\prime}$, if for a third proper representative $\left(\bar{f}, \bar{S}, \overline{\mathcal{V}}, \bar{D}_{A}\right)$ of $A$ fitting into the commutative squares (3.8) so that

$$
\left(\left.g^{\prime}\right|_{\bar{D}_{A}}\right)^{*}\left(h_{f^{\prime}}^{\prime}\right)=\left(\left.g\right|_{\bar{D}_{A}}\right)^{*}\left(h_{f}\right) \in \mathbf{k}\left(\bar{D}_{A}\right)
$$

We define a rational function on $A$ to be an equivalence class of proper representatives of rational functions of $A$.

We define an integral rational equivalence be a pair $(A, h)$ of an integral cycle $A$ of $\mathcal{F}$ and a non-trivial rational function $h$ on $A$. We define $W_{*} \mathcal{F}$ be the (rational) linear combinations of integral rational equivalences of $\mathcal{F}$. We now define the boundary homomorphism

$$
\partial: W_{*} \mathcal{F} \rightarrow Z_{*} \mathcal{F}
$$

Let $(A, h)$ be a rational equivalence with $A$ integral and with a proper presentation $\left(f, S, \mathcal{V}, D_{A}\right)$ as in (3.6) and $h_{f} \in \mathbf{k}\left(D_{A}\right)^{*}$. We now construct $\partial(A, h)$. We first express

$$
\partial\left(D_{A}, h_{f}\right)=\sum_{A \in I} n_{a}\left[D_{a}\right],
$$

where $D_{a} \subset \mathcal{V}$ are integral. Let $\zeta_{S}: \mathcal{V} \rightarrow f^{*} \mathcal{F}$ be the tautological map and let $\zeta_{X}: f^{*} \mathcal{F} \rightarrow \mathcal{F}$ be the projection. By our definition of $h_{f}$, for each $D_{a}$, the 
$B_{a, S}=\zeta_{S}\left(D_{a}\right) \subset f^{*} \mathcal{F}$ has the property that $D_{a}=\zeta_{S}^{-1}\left(B_{a, S}\right)$. Hence following the proof of Proposition 2.1, one checks that $B_{a, S}$ is an integral cycle. We let $B_{a}=\zeta_{X}\left(B_{a, S}\right) \subset \mathcal{F}$; it is an integral cycle in $\mathcal{F}$. Let $e=\operatorname{deg} f$; after defining $e_{a}$, which should be the degree of $\left.\zeta_{X}\right|_{B_{a, S}}: B_{a, S} \rightarrow B_{a}$, we define

$$
\partial(A, h)=e^{-1} \sum_{a \in I} n_{a} e_{a}\left[B_{a}\right] .
$$

We define the degree $e_{a}$ using étale representative of $(A, h)$. We let $\pi$ : $\mathcal{F} \rightarrow X$ be the projection. Since $B_{a} \subset \mathcal{F}$ is an integral cycle, $\pi\left(B_{a}\right) \subset Y \subset X$ is an integral substack. We pick an affine $S^{\prime}$ and an étale $f^{\prime}: S^{\prime} \rightarrow Y$ so that $S^{\prime} \times_{Y} \pi\left(B_{a}\right) \rightarrow \pi\left(B_{a}\right)$ is dominant. We pick a locally free sheaf $\mathcal{V}^{\prime}$ on $S^{\prime}$ and a surjective $V^{\prime} \rightarrow f^{\prime *} \mathcal{F}$. By shinking $S^{\prime}$ if necessary, we can assume $\operatorname{rank} \mathcal{V}=\operatorname{rank} \mathcal{V}^{\prime}$. We let $D_{A}^{\prime}=A \times_{\mathcal{F}} \mathcal{V}^{\prime} \subset \mathcal{V}^{\prime}$.

To compare $D_{A}$ and $D_{A}^{\prime}$, we form $T=S \times_{Y} S^{\prime}$; we let $p: T \rightarrow S$ and $p^{\prime}$ : $T \rightarrow S^{\prime}$ be the projections. We then pick an affine opens $U \subset T$ so that if we let $\varphi: \mathcal{V} \rightarrow S$ be the projection, $U \cap\left(\varphi\left(D_{a}\right) \times_{Y} S^{\prime}\right)$ is dense in $\varphi\left(D_{a}\right) \times_{Y} S^{\prime}$; we pick a dense affine open $U_{0} \subset U$ so that $\left.p^{\prime}\right|_{U_{0}}: U_{0} \rightarrow S^{\prime}$ is étale. Since $U$ is affine, we can find an isomorphism $\left.\left.p^{*} \mathcal{V}\right|_{U} \cong p^{* *} \mathcal{V}^{\prime}\right|_{U}$ that commutes with the projection $p^{*} \mathcal{V} \rightarrow p^{*} f^{*} \mathcal{F}$ and $p^{*} \mathcal{V}^{\prime} \rightarrow p^{*} f^{*} \mathcal{F}$ :
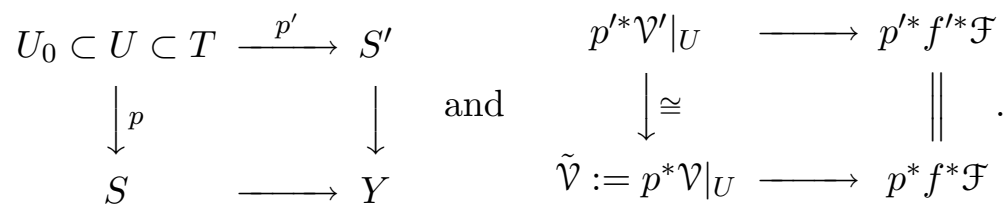

We now view $D_{A} \times{ }_{S} U$ and $D_{A}^{\prime} \times{ }_{S^{\prime}} U$ as subsets in $\tilde{\mathcal{V}}$ using the isomorphisms above, and using that $U_{0} \rightarrow Y$ is étale. Since $U_{0} \rightarrow Y$ is étale, we have that

$$
D_{A} \times_{S} U_{0}=D_{A}^{\prime} \times_{S^{\prime}} U_{0}=A \times\left.\left.\mathcal{F} \tilde{\mathcal{V}}\right|_{U_{0}} \subset \tilde{\mathcal{V}}\right|_{U_{0}}
$$

We let $\tilde{D}_{A}$ be the closure of $D_{A} \times{ }_{S} U_{0}$ in $\tilde{\mathcal{V}}$. We let $q: \tilde{\mathcal{V}} \rightarrow \mathcal{V}$ and $q^{\prime}: \tilde{\mathcal{V}} \rightarrow \mathcal{V}^{\prime}$ be the projections.

By our assumption on $h_{f} \in \mathbf{k}\left(D_{A}\right)^{*}$, we see that $\left(\left.q\right|_{\tilde{D}_{A}}\right)^{*}\left(h_{f}\right)$ descends to a rational function on $D_{A}^{\prime}$; we denote the descent by $h_{f^{\prime}} \in \mathbf{k}\left(D_{A}^{\prime}\right)^{*}$. We express

$$
\partial\left(D_{A}^{\prime}, h_{f^{\prime}}\right)=\sum_{b \in I^{\prime}} n_{b}^{\prime}\left[D_{b}^{\prime}\right]
$$

where $D_{b}^{\prime}$ are integral. 
Finally, we pick a $b(a) \in I^{\prime}$ so that the intersection

$$
\tilde{D}_{a}:=\left(D_{a} \times{ }_{S} U\right) \cap\left(D_{b(a)}^{\prime} \times{ }_{S^{\prime}} U\right) \subset \tilde{\mathcal{V}}
$$

where we view both $D_{a} \times{ }_{S} U$ and $D_{b(a)}^{\prime} \times{ }_{S^{\prime}} U$ as subsets of $\tilde{\mathcal{V}}$ using the isomorphisms in the square above, $\tilde{D}_{a}$ dominates both $D_{a}$ and $D_{b(a)}^{\prime}$. Because $h_{f^{\prime}}$ is the descent of $\left(\left.q\right|_{\tilde{D}_{A}}\right)^{*}\left(h_{f}\right)$, a direct checking shows that such $b(a) \in I^{\prime}$ exists.

The geometric meaning of this construction mimics the fiber product over $\mathcal{F}$. We let $\left.\bar{B}_{a} \subset \mathcal{F}\right|_{S}$ be the image stack of $D_{a}$ under $\mathcal{V} \rightarrow f^{*} \mathcal{F}=\left.\mathcal{F}\right|_{S}$. We let $\left.B_{b(a)}^{\prime} \subset \mathcal{F}\right|_{S^{\prime}}$ be the image of $D_{b(a)}^{\prime}$ under $\mathcal{V}^{\prime} \rightarrow f^{\prime *} \mathcal{F}=\left.\mathcal{F}\right|_{S^{\prime}}$. Our assumption that (3.12) dominates $D_{a}$ and $D_{b(a)}^{\prime}$ ensures that the image of $B_{b(a)}^{\prime}$ under $\left.\mathcal{F}\right|_{S^{\prime}} \rightarrow \mathcal{F}$ is $B_{a}$. (Note that $\bar{B}_{a}$ maps to $B_{a}$ because $B_{a}$ is the image of $D_{a}$.) This way, the degree of $\bar{B}_{a} \rightarrow B_{a}$ is the same as the degree of

$$
\bar{B}_{a} \times_{\mathcal{F}} B_{b(a)}^{\prime} \longrightarrow B_{b(a)}^{\prime}
$$

To define this degree, we pull back the first term in (3.13) to the bundle $\tilde{\mathcal{V}}$ over $U$ to obtain the $\tilde{D}_{a}$ in (3.12); we pull back the second term in (3.13) to $\mathcal{V}^{\prime}$ to obtain $D_{b(a)}^{\prime} \subset \mathcal{V}^{\prime}$. Thus the degree of (3.13) is the same as

$$
e_{a}:=\operatorname{deg}\left(\left.q^{\prime}\right|_{\tilde{D}_{a}}: \tilde{D}_{a} \longrightarrow D_{b(a)}^{\prime}\right)
$$

Since this definition uses the fact that the degree of a map is preserved after an étale base change of both the domain and the target, it implies that $e_{a}$ is well-defined, independent of the choice of the étale cover $S^{\prime} \rightarrow Y$ we pick. Since the checking is routine, we omit it here.

With $e_{a}$ defined, we define $\partial(A, h)$ using (3.11). By linear extension, we obtain the boundary operation (3.9).

Corollary 3.1. We have the relation

$$
s^{!} \circ \partial=0: W_{*} \mathcal{F} \longrightarrow A_{*} X .
$$

Proof. We only need to check that for any integral $(A, h) \in W_{*} \mathcal{F}$, we have $(s ! \circ \partial)(A, h)=0$. The proof is routine using proper representative of $(A, h)$, which transform this identity to the identity $s^{!} \circ \partial$ for rational equivalence in a vector bundle over a scheme. Since the proof follows the argument in [11] and $[6$, Sect. 3], we will omit the details here. 
Definition-Theorem 3.1. Let $\mathcal{M}$ be an Artin stack locally of finite type, and let $X$ be a proper Deligne-Mumford stack of finite presentation. Suppose $\mathcal{M}$ is smooth and of pure dimension, and suppose $\phi$ is a semi-perfect relative obstruction theory of $X / \mathcal{M}$. The stated procedure produces a (virtual normal) cone cycle $\left[\mathfrak{c}_{X / \mathcal{M}}\right] \in Z_{*} \mathcal{O} b_{\phi}$. We define the virtual cycle of $X$ be

$$
[X, \phi]^{\text {vir }}:=s^{!}\left[\mathfrak{c}_{X / \mathcal{M}}\right] \in A_{*} X .
$$

We prove that the virtual cycle $\left[X, \phi_{\bullet}\right]^{\text {vir }}$ is deformation invariant in the sense of cycles. Consider a fiber-diagram [2, Sect. 7] of separated DeligneMumford stacks $X$ and $X^{\prime}$ to smooth Artin stacks $\mathcal{M}$ and $\mathcal{M}^{\prime}$

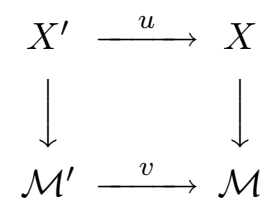

such that $\mathcal{M}$ and $\mathcal{M}^{\prime}$ are of pure dimensions, $u$ is representable, and $v$ : $\mathcal{M}^{\prime} \rightarrow \mathcal{M}$ is a regular immersion. Let $v^{!}: A_{*} X \rightarrow A_{*} X^{\prime}$ be the Gysin homomorphism associated to this square.

Proposition 3.2. A semi-perfect relative obstruction theory $\phi$ of $X / \mathcal{M}$ induces a semi-perfect relative obstruction theory $\phi^{\prime}$ of $X^{\prime} / \mathcal{M}^{\prime}$, and their virtual cycles are related by

$$
v^{!}[X, \phi]^{\mathrm{vir}}=\left[X^{\prime}, \phi^{\prime}\right]^{\mathrm{vir}}
$$

Proof. We let $\phi$ be given by $\left\{\phi_{\alpha}, U_{\alpha}, E_{\alpha}, \psi_{\alpha \beta}\right\}_{\Lambda}$. We cover $X^{\prime}$ by $U_{\alpha}^{\prime}=$ $U_{\alpha} \times_{X} X^{\prime}$. Let $u_{\alpha}$ be the induced morphism fitting into the Cartesian square

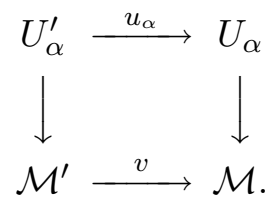

For each $\alpha \in \Lambda$, we let

$$
\phi_{\alpha}^{\prime}=u_{\alpha}^{*} \phi_{\alpha}: E_{\alpha}^{\prime}:=u_{\alpha}^{*} E_{\alpha} \longrightarrow u_{\alpha}^{*} L_{U_{\alpha} / \mathcal{M}} \longrightarrow L_{U_{\alpha}^{\prime} / \mathcal{M}^{\prime}},
$$

where the last arrow is induced from the Cartesian square (3.14).

According to [2, Prop. 7.2], $\phi_{\alpha}^{\prime}$ is a perfect obstruction theory of $U_{\alpha}^{\prime} \rightarrow$ $\mathcal{M}^{\prime}$. To form a semi-perfect obstruction theory of $X^{\prime} \rightarrow \mathcal{M}^{\prime}$, we need transitions $\psi_{\alpha \beta}^{\prime}$. Over $U_{\alpha \beta}^{\prime}=U_{\alpha}^{\prime} \times_{X^{\prime}} U_{\beta}^{\prime}=X^{\prime} \times_{X} U_{\alpha \beta}$, let $u_{\alpha \beta}: U_{\alpha \beta}^{\prime} \rightarrow U_{\alpha \beta}$ be 
the tautological inclusion. We define

$$
\psi_{\alpha \beta}^{\prime}:=\left(u_{\alpha \beta}\right)^{*}\left(\psi_{\alpha \beta}\right):\left.\left.E_{\alpha}^{\prime}\right|_{U_{\alpha \beta}^{\prime}} \longrightarrow E_{\beta}^{\prime}\right|_{U_{\alpha \beta}^{\prime}} .
$$

Since $\phi$ is a semi-perfect obstruction theory, one checks that $\phi^{\prime}=\left\{U_{\alpha}^{\prime}, \phi_{\alpha}^{\prime}\right.$, $\left.E_{\alpha}^{\prime}, \psi_{\alpha \beta}^{\prime}\right\}$ is a semi-perfect obstruction theory of $X^{\prime} / \mathcal{M}^{\prime}$. Since the checking is routine, we will omit the detail.

We now prove the identity $v^{!}[X, \phi]^{\text {vir }}=\left[X^{\prime}, \phi^{\prime}\right]^{\text {vir }}$. Let $L=N_{\mathcal{M}^{\prime} / \mathcal{M}}$ be the normal bundle to $\mathcal{M}^{\prime}$ in $\mathcal{M}$. Because $v: \mathcal{M}^{\prime} \rightarrow \mathcal{M}$ is a regular closed immersion, $V$ is a vector bundle. By the proof of [2, Prop. 7.2] and [7], the Vistoli's rational equivalence

$$
(R, h) \in W_{*}\left(L \times\left.{ }_{\mathcal{M}} \mathcal{C}_{X / \mathcal{M}}\right|_{X^{\prime}}\right) \subset W_{*}\left(L \times\left.\mathcal{M}^{\prime} N_{X / \mathcal{M}}\right|_{X^{\prime}}\right)
$$

gives the following identity as cycles:

$$
\partial(R, h)=\left[\mathcal{C}_{\left.\mathcal{C}_{X / \mathcal{M}}\right|_{X^{\prime}} / \mathcal{C}_{X / \mathcal{M}}}\right]-\left[L \times \mathcal{M}^{\prime} \mathcal{C}_{X^{\prime} / \mathcal{M}^{\prime}}\right] \in Z_{*}\left(L \times\left._{\mathcal{M}^{\prime}} N_{X / \mathcal{M}}\right|_{X^{\prime}}\right) .
$$

In case $X / \mathcal{M}$ has a perfect relative obstruction theory, push-forward this relation to the bundle-stack of the obstruction complex proves the desired identity $v^{!}[X, \phi]^{\text {vir }}=\left[X^{\prime}, \phi^{\prime}\right]^{\text {vir }}$.

We now show that $(3.15)$ proves the same identity in the case of semiperfect obstruction theory. We let

$$
\mathcal{F}:=L \times\left._{\mathcal{O}_{\mathcal{M}}} \mathcal{O} b_{X / \mathcal{M}}\right|_{X^{\prime}}
$$

which is $L \times{ }_{\mathcal{O}_{\mathcal{M}}^{\prime}} \mathcal{O} b_{X^{\prime} / \mathcal{M}^{\prime}}$ since $\mathcal{O} b_{X^{\prime} / \mathcal{M}^{\prime}}=\left.\mathcal{O} b_{X / \mathcal{M}}\right|_{X^{\prime}}$.

We let

$$
\eta_{\alpha}: L \times\left.\left.\mathcal{M}^{\prime} N_{X / \mathcal{M}}\right|_{U_{\alpha}^{\prime}} \longrightarrow \mathcal{F}\right|_{U_{\alpha}^{\prime}}=L \times \mathcal{M}^{\prime} \mathcal{O} b_{U_{\alpha}^{\prime} / \mathcal{M}^{\prime}}
$$

be the morphism (of stacks) induced by $\left(\mathbb{L}_{U_{\alpha} / \mathcal{M}}^{\geq-1}\right)^{\vee} \rightarrow E_{\alpha}^{\vee} \rightarrow H^{1}\left(E_{\alpha}^{\vee}\right)$. Repeating the proof of Proposition 2.1, we conclude that the collection of image cycles $\eta_{\alpha *}\left[L \times \mathcal{M}^{\prime} \mathcal{C}_{X^{\prime} / \mathcal{M}^{\prime}}\right]$ (resp. $\left.\eta_{\alpha *}\left[\left.\mathcal{C}_{\left.\mathcal{C}_{X / \mathcal{M}}\right|_{X^{\prime}} / \mathcal{C}_{X / \mathcal{M}}}\right|_{U_{\alpha}^{\prime}}\right]\right)$ forms a cycle in $\mathcal{F}$. Obviously, the former form the cycle $L \times \mathcal{M}^{\prime} \mathfrak{c}_{X^{\prime}} / \mathcal{M}^{\prime}$; we denote the later by $[\mathfrak{b}] \in Z_{*} \mathcal{F}$. Letting $s_{0}$ be the zero section of the stack $\mathcal{O} b_{X^{\prime} / \mathcal{M}^{\prime}}$, we conclude

$$
s^{!}\left[L \times \mathcal{M}^{\prime} \mathfrak{c}_{X^{\prime} / \mathcal{M}^{\prime}}\right]=s_{0}^{!}\left[\mathfrak{c}_{X^{\prime}} / \mathcal{M}^{\prime}\right]=\left[X^{\prime}, \phi^{\prime}\right]^{\text {vir }} \in A_{*} X^{\prime} .
$$

Mimic the proof given in [11] and [6, Sect. 3], we conclude that

$$
s^{!}[\mathfrak{b}]=v^{!}[X, \phi]^{\text {vir }} \in A_{*} X^{\prime} .
$$


Finally, like before we can push $R$ via $\eta_{\alpha *}$ to form a cycle in $\mathcal{F}$; we then check that $h$ descends to a rational function on this cycle, resulting a rational equivalence $\beta \in W_{*} \mathcal{F}$. Then the relation (3.15) gives

$$
\partial \beta=[\mathfrak{b}]-\left[L \times_{\mathcal{M}^{\prime}} \mathfrak{c}_{X^{\prime} / \mathcal{M}^{\prime}}\right] \in Z_{*} \mathcal{F} .
$$

Since the argument is routine, we will omit the details here.

Combined, we have

$$
v^{!}[X, \phi]^{\mathrm{vir}}=s^{!}\left[L \times \mathcal{M}^{\prime} \mathfrak{c}_{X^{\prime} / \mathcal{M}^{\prime}}\right]=s^{!}[\mathfrak{b}]-s^{!}(\partial \beta)=\left[X^{\prime}, \phi^{\prime}\right]^{\mathrm{vir}} .
$$

This proves the theorem.

\section{Virtual cycle of derived objects and deformation invariance}

In this section, we construct semi-perfect obstruction theory of the moduli of derived objects on a projective Calabi-Yau threefold. In case the moduli space has an open, proper Deligne-Mumford substack, the virtual class of the semi-perfect obstruction defines the Donaldson-Thomas invariant of this moduli space. This for instance applies to the moduli spaces constructed in [15].

We fix a smooth family of projective Calabi-Yau threefolds $S \rightarrow B$. We follow the convention that for $T \rightarrow B$, we use $p_{S}$ and $p_{T}$ to denote the projections of $S \times{ }_{B} T$ to $S$ and $T$.

We quote a theorem of Inaba [5], generalized by Lieblich [14].

Theorem 4.1. Let $\mathfrak{D}_{S / B}$ be the stack of objects $E$ in $D^{b}(S)$ that are relatively perfect over $B$ and that for all geometric points $b \in B$ we have $\operatorname{Ext}^{i<0}$ $\left(E_{b}, E_{b}\right)=0$ and $\operatorname{Ext}^{0}\left(E_{b}, E_{b}\right)=\mathbf{k}(b)$. Then $\mathfrak{D}_{S / B}$ is a Deligne-Mumford stack locally of finite presentation over $B$.

Recall that since $S \rightarrow B$ is smooth and projective, an $E \in D^{b}\left(S \times_{B} T\right)$ is relatively perfect if it is locally perfect [14]. In this section, we are interested in the substack of derived objects with fixed determinant line bundle.

Definition 4.1. Let $E \in D^{b}\left(S \times_{B} T\right)$ be relatively perfect, and let $L$ be a line bundle on $S$. We say $\operatorname{det} \mathcal{E} \sim p_{S}^{*} L$ if there is a line bundle $J$ on $T$ such that $\operatorname{det} E \cong p_{S}^{*} L \otimes p_{T}^{*} J$. 
Following Inaba [5], we introduce the moduli functor

$$
\operatorname{Splcpx}_{S / B}^{L}:(\operatorname{Sch} / B) \longrightarrow(\operatorname{Sets})
$$

that sends any $B$-scheme $T$ to the set of all $E \in D^{b}\left(S \times_{B} T\right)$ satisfying the requirements in Theorem 4.1 and $\operatorname{det} E \sim p_{S}^{*} L$. Applying the proof in [5] (see also [14]), its étale sheafification $\mathfrak{D}_{S / B}^{L}$ is a Deligne-Mumford stack, locally of finite presentation over $B$.

We introduce the notion of semi-families and show that $\mathfrak{D}_{S / B}^{L}$ admits a universal semi-family.

Definition 4.2. For any Deligne-Mumford stack $X \rightarrow B$, a semi-family of derived objects in $D^{b}(S)$ on $X$ consists of an étale open covering $\left\{U_{\alpha}\right\}_{\Lambda}$ of $X$, derived objects $E_{\alpha} \in D^{b}\left(S \times_{B} U_{\alpha}\right)$, and quasi-isomorphisms

$$
f_{\alpha \beta}:\left.\left.E_{\alpha}\right|_{S \times{ }_{B} U_{\alpha \beta}} \cong E_{\beta}\right|_{S \times{ }_{B} U_{\alpha \beta}} \quad \text { in } \quad D^{b}\left(S \times_{B} U_{\alpha \beta}\right),
$$

that satisfy the semi-cocycle condition: for any triple $(\alpha, \beta, \gamma)$ in $\Lambda$, there is a $c_{\alpha \beta \gamma} \in \Gamma\left(\mathcal{O}_{U_{\alpha \beta \gamma}}^{*}\right)$ such that

$$
\pi_{\gamma \alpha}^{*} f_{\gamma \alpha} \circ \pi_{\beta \gamma}^{*} f_{\beta \gamma} \circ \pi_{\alpha \beta}^{*} f_{\alpha \beta}=c_{\alpha \beta \gamma} \cdot \mathrm{id}:\left.\left.E_{\alpha}\right|_{S \times_{B} U_{\alpha \beta \gamma}} \longrightarrow E_{\alpha}\right|_{S \times_{B} U_{\alpha \beta \gamma}},
$$

where $\pi_{\alpha \beta}: S \times_{B} U_{\alpha \beta \gamma} \rightarrow S \times{ }_{B} U_{\alpha \beta}$ is the tautological projection.

Definition 4.3. Let $X \subset \mathfrak{D}_{S / B}^{L}$ be an open substack. A universal semifamily over $X$ consists of a semi-family $\left\{E_{\alpha}, f_{\alpha \beta}\right\}_{\Lambda}$ on $X$ of which the following holds: for any scheme $T$ over $B$ and any object $F \in \operatorname{Splcpx}_{S / B}^{L}(T)$ such that the induced morphism $T \rightarrow \mathfrak{D}_{S / B}^{L}$ (induced by $F$ ) factors through $X \subset \mathfrak{D}_{S / B}^{L}$, then there is an étale cover $\left\{T_{\alpha}\right\}_{\Lambda}$ of $T$ (indexed by the same $\Lambda$ in $\left.\left\{U_{\alpha}\right\}_{\Lambda}\right)$ and morphisms $\varphi_{\alpha}: T_{\alpha} \rightarrow U_{\alpha}$ such that

$$
\left.\left(1_{S} \times \varphi_{\alpha}\right)^{*} E_{\alpha} \cong F\right|_{S \times_{B} U_{\alpha}}
$$

Proposition 4.1. Let $X \subset \mathfrak{D}_{S / B}^{L}$ be an open and closed substack. Then $X$ admits a universal semi-family.

Proof. The proof follows from that $\mathfrak{D}_{S / B}^{L}$ is a sheafification of $\operatorname{Splcpx}{ }_{S / B}^{L}$.

Using universal semi-families of $X$, and applying the Atiyah class constructed by Huybrecht-Thomas [3], we construct semi-perfect obstruction theory of $X / B$. 
Lemma 4.1. Let $X \subset \mathfrak{D}_{S / B}^{L}$ be an open substack. Then $X \rightarrow B$ has a semiperfect relative obstruction theory given by the Atiyah class constructed in [3].

Proof. Let $\left\{E_{\alpha}, f_{\alpha \beta}\right\}_{\Lambda}$ be a universal semi-family. Using that it is universal locally, we conclude that for any closed $x \in U_{\alpha}$, the family $E_{\alpha}$ restricted to the formal completion of $U_{\alpha}$ at $x$ is the universal family of the hull of the infinitesimal deformations of $x$ in $U_{\alpha}$. Thus applying the work of Huybrecht-Thomas [3], after fixing a closed embedding $U_{\alpha} \rightarrow W_{\alpha}$ into a $B$-smooth scheme $W_{\alpha}$ and presenting the truncated cotangent complex of $U_{\alpha} \rightarrow B$ as

$$
L_{U_{\alpha} / B}=\left[I_{U_{\alpha} \subset W_{\alpha}} /\left.I_{U_{\alpha} \subset W_{\alpha}}^{2} \rightarrow \Omega_{W_{\alpha} / B}\right|_{U_{\alpha}}\right]
$$

we obtain a perfect relative obstruction theory

$$
\phi_{\alpha}: F_{\alpha}:=\operatorname{RHom}_{\mathcal{O}_{U_{\alpha}}}\left(E_{\alpha}, E_{\alpha}\right)_{0} \longrightarrow L_{U_{\alpha} / B}
$$

where the subscript 0 stands for the traceless part.

Using $f_{\alpha \beta}$, we obtain an isomorphism $g_{\alpha \beta}$ as shown making the square

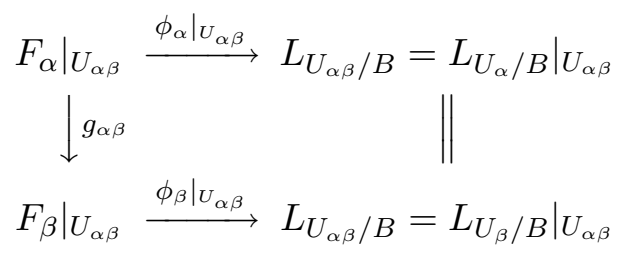

commutative in $D\left(U_{\alpha \beta}\right)$. Let

$$
\psi_{\alpha \beta}:=H^{1}\left(g_{\beta \alpha}^{\vee}\right):\left.\left.H^{1}\left(F_{\alpha}^{\vee}\right)\right|_{U_{\alpha \beta}} \longrightarrow H^{1}\left(F_{\beta}^{\vee}\right)\right|_{U_{\alpha \beta}} .
$$

Because a scaling automorphism $c_{\alpha} \cdot$ id $: E_{\alpha} \rightarrow E_{\alpha}, c_{\alpha} \in \Gamma\left(\mathcal{O}_{U_{\alpha}}^{*}\right)$, induces the identity automorphism of $\operatorname{RHom}_{\mathcal{O}_{U_{\alpha}}}\left(E_{\alpha}, E_{\alpha}\right)_{0}$, the cocycle condition (4.1) implies that for any triple indices $(\alpha, \beta, \gamma)$, pullback to $U_{\alpha \beta \gamma}$ we obtain identity

$$
\left.\left.\left.\psi_{\gamma \alpha}\right|_{U_{\alpha \beta \gamma}} \circ \psi_{\beta \gamma}\right|_{U_{\alpha \beta \gamma}} \circ \psi_{\alpha \beta}\right|_{U_{\alpha \beta \gamma}}=\mathrm{id} \text {. }
$$

This proves that $\left\{U_{\alpha}, \phi_{\alpha}, E_{\alpha}, \psi_{\alpha \beta}\right\}_{\Lambda}$ is a semi-perfect relative obstruction theory of $X \rightarrow \mathcal{M}$.

Definition-Theorem 4.1. In case $B$ is a closed point, $S$ is a smooth projective Calabi-Yau threefold and $L$ is a line bundle on $S$, for any proper, open 
and closed substack $X \subset \mathfrak{D}_{S}^{L}$, we define its associated Donaldson-Thomas invariant be $\operatorname{deg}[X]^{\text {vir }}$, where $[X]^{\text {vir }}$ is the virtual cycle constructed by applying Definition-Theorem 3.1 to the semi-perfect obstruction theory of $X$ constructed in Lemma 4.1.

Back to the case of a smooth family of projective Calabi-Yau $S \rightarrow B$ over a smooth base $B$, we let $X \subset \mathfrak{D}_{S / B}^{L}$ be a $B$-proper, open and closed substack. For any closed point $b \in B$, we denote $X_{b}=X \times_{B} b$, denote $i_{b}: X_{b} \rightarrow X$ the inclusion and $i_{b}^{!}: A_{*} X \rightarrow A_{*} X_{b}$ the Gysin map.

Corollary 4.1. Let the situation be as stated, then $i_{b}^{!}[X]^{\mathrm{vir}}=\left[X_{b}\right]^{\mathrm{vir}}$.

Proof. This follows from Proposition 3.2.

This confirms the deformation invariance of Donaldson-Thomas invariant of proper moduli of derived objects.

\section{Further comments}

One can define the same Donaldson-Thomas invariant of a moduli of derived objects using Behrend's weighted Euler number [1], based on HuybrechtThomas' construction of perfect obstruction theory [3]. However, semiperfect obstruction theory proves that the Donaldson-Thomas invariant of derived objects is deformation invariant.

We can weaken the assumption on semi-perfect obstruction theory by replacing (1) in Definition 3.1 by that restricting $\mathcal{O} b_{\phi_{\alpha}}$ to the reduced part $\left(U_{\alpha}\right)_{\text {red }}$ of $U_{\alpha}$ descend to a sheaf on the reduced part of $X$; the item $(2)$ is unchanged since $\nu$-equivalent only requires the restriction of the obstruction sheaves to the reduced part of the Deligne-Mumford stack. The results of this paper hold true in this weaker version of semi-perfect obstruction theory. Since we do not see immediate application of this, for notational simplicity, in the end we phrase the semi-perfect obstruction theory relying on the full obstruction sheaf of the stack.

In many applications, the $\psi_{\alpha \beta}$ in (3.1) are induced from quasiisomorphisms

$$
\Psi_{\alpha \beta}:\left.\left.E_{\alpha}\right|_{U_{\alpha \beta}} \longrightarrow E_{\beta}\right|_{U_{\alpha \beta}} .
$$

If we further assume that these quasi-isomorphisms form a descent data for the stacks $h^{1} / h^{0}\left(E_{\alpha}^{\vee}\right)$, namely there is a two-term perfect complex $E$ on $X$ such that $\left.E_{\alpha} \cong E\right|_{U_{\alpha}}$ and the quasi-isomorphism $\Psi_{\alpha \beta}$ is isomorphic 
to that induced by the identity map of $E$, then we can use intersection theory on bundle stacks to define the virtual cycles. Indeed, by assumption, $\mathcal{O} b_{\phi} \cong H^{1}\left(E^{\vee}\right)$, which induces the coarse moduli functor $h^{1} / h^{0}\left(E^{\vee}\right) \rightarrow \mathcal{O} b_{\phi}$. For the cycle $\left[\mathfrak{c}_{X / \mathcal{M}}\right] \in Z_{*} \mathcal{O} b_{\phi}$, by expressing it as rational combination of integral cycles $\left[\mathfrak{c}_{X / \mathcal{M}}\right]=\sum_{i} n_{i}\left[\mathfrak{c}_{i}\right]$, we define $\tilde{\mathfrak{c}}_{i}=\mathfrak{c}_{i} \times \mathcal{O}_{\phi} h^{1} / h^{0}\left(E^{\vee}\right)$, where each $\tilde{\mathfrak{c}}_{i} \in Z_{*} h^{1} / h^{0}\left(E^{\vee}\right)$ is an integral cycle, and define

$$
\left[\tilde{\mathfrak{c}}_{X / \mathcal{M}}\right]=\sum_{i} n_{i}\left[\tilde{\mathfrak{c}}_{i}\right] \in Z_{*} h^{1} / h^{0}\left(E^{\vee}\right)
$$

Let $\tilde{s}$ be the zero section of $h^{1} / h^{0}\left(E^{\vee}\right)$; using the Gysin map $\tilde{s}^{!}$defined in [9], we obtain

$$
\tilde{s}^{!}\left[\tilde{\mathfrak{c}}_{X / \mathcal{M}}\right]=s^{!}\left[\mathfrak{c}_{X / \mathcal{M}}\right] \in A_{*} X
$$

This way, the intersection theory on Artin stacks can be applied directly.

The last comment is on the relation of the perfect obstruction theory formulated by Tian and the second named author in [13] with the semiperfect obstruction theory defined in this paper. It can be shown that a "family Kuranishi model" over an affine chart $U_{\alpha} \rightarrow X$ constructed in [13] induces a perfect obstruction $\phi_{\alpha}: E_{\alpha} \rightarrow L_{U_{\alpha} / \mathcal{M}}$ as formulated in [2]. The $\nu$-equivalence over intersection $U_{\alpha \beta}$ follows from the definition of the perfect obstruction theory in [13]. It is in this sense we say that the semi-perfect obstruction theory is a mixture of the two versions of perfect obstruction theories formulated in $[2,13]$.

\section{Acknowledgments}

We thank S. Katz for pointing out an oversight of the original version of this paper. The first named author is supported by a Hong Kong DAG grant. The second named author is partially supported by an NSF grant and a DARPA grant.

\section{References}

[1] K. Behrend, Symmetric obstruction theories and Hilbert schemes of points on threefolds, Algebra Number Theory 2(3) (2008), 313-345.

[2] K. Behrend and B. Fantechi, The intrinsic normal cone, Invent. Math. 128(1) (1997), 45-88. 
[3] D. Huybrechts and R.P. Thomas, Deformation-obstruction theory for complexes via Atiyah and Kodaira Spencer classes, Math. Ann. 346 (2010), 545-569.

[4] L. Illusie, Complexe cotangent et deformations I,II, in 'Lecture Notes in Mathematics', 239, 283, Springer, Berlin, Heidelberg, New York, 1971.

[5] M. Inaba, Toward a definition of moduli of complexes of coherent sheaves on a projective scheme, J. Math. Kyoto Univ. 42(2) (2002), 317-329.

[6] Y.H. Kiem and J. Li, Localized virtual cycle by cosections, preprint, arxiv: 1007.3085.

[7] B. Kim, A. Kresch and T. Pantev, Functoriality in intersection theory and a conjecture of Cox, Katz, and Lee, J. Pure Appl. Algebra 179(1-2) (2003), 127-136.

[8] A. Kresch, Canonical rational equivalence of intersections of divisors, Invent. Math. 136(3) (1999), 483-496.

[9] A. Kresch, Cycle groups for Artin stacks, Invent. Math. 138(3) (1999), 495-536.

[10] G. Laumon and L. Moret-Bailly, Champs algébriques, in 'A series of Modern Surveys in Mathematics', Springer-Verlag, Berlin, 2000.

[11] J. Li, A degeneration formula of $G W$-invariants, J. Differential Geom. 60(2) (2002), 199-293.

[12] J. Li, Zero dimensional Donaldson-Thomas invariants of threefolds, Geom. Topol. 10 (2006), 2117-2171.

[13] J. Li and Gang. Tian, Virtual moduli cycles and Gromov-Witten invariants of algebraic varieties, J. Amer. Math. Soc. 11(1) (1998), 119-174.

[14] M. Lieblich, Moduli of complexes on a proper morphism, J. Alebraic Geom. 15(1) (2006), 175-206.

[15] J. Lo, Moduli of PT-semistable objects II, arXiv:1011.6306.

[16] A. Vistoli, Intersection theory on algebraic stacks and on their moduli spaces, Invent. Math. 97 (1989), 613-670. 
Department of Mathematics

Hong Kong University of Science and Technology

HONG KONG

E-mail address: mahlchang@ust.hk

Department of Mathematics

STANFORD UNIVERSITY

STANFORD, CA 94305

USA

E-mail address: jli@math.stanford.edu

ReCEIVED June 23, 2011 